\section{Responsabilidad social empresarial e integración laboral de personas con discapacidad intelectual. Estudio de caso}

\author{
Corporate social responsibility and \\ integration of people with intellectual \\ disabilities. Case study
}

Palabras clave

Responsabilidad social empresarial, discapacidad intelectual, empleo con apoyo, preparador laboral.

\section{Keywords}

Corporate social responsibility, intellectual disabilities, supported employment, job coach.

\section{Introducción}

Este ensayo es fruto del análisis de diez años de trabajo en la integración laboral de personas con discapacidad intelectual. Desde 2005 el Grupo Alimerka gestiona un programa de empleo con apoyo que ha sido reconocido en 20I 3 por la Fundación ONCE con el Premio Solidarios ${ }^{\mathrm{T}}$. Los datos de las evaluaciones anuales de este programa nos permiten presentar algunas reflexiones sobre el empleo en entornos normalizados.
I. Además del Premio Solidarios otorgado en 2013 por la Fundación ONCE al Grupo Alimerka por la labor de integración social y laboral de personas con discapacidad, el programa de empleo con apoyo coordinado por la Fundación Alimerka ha recibido los siguientes reconocimientos: Reconocimiento especial por la colaboración en beneficio de las personas con discapacidad, otorgado por la Asociación Emburria de Cangas de Onís (20I2); Premio de la Confederación Española de Personas con Discapacidad Física y Orgánica (COCEMFE Asturias) por la integración laboral de personas con discapacidad (20I0); Premio de la Federación de Asociaciones de Familiares y Personas con Enfermedad Mental de Castilla y León, a la responsabilidad social corporativa (2008); y Premio Martillo Solidario - Rompiendo Barredas de la Asociación Down de León (2006).

\section{Antonio Blanco Prieto}

<antonioblanco@fundacionalimerka.es>

Fundación Alimerka. Universidad de Oviedo

\section{Para citar:}

Blanco, A. (20I 5): "Responsabilidad social empresarial e integración laboral de personas con discapacidad intelectual. Estudio de caso", Revista Española de Discapacidad, 3 (I): 2II-2I7.

Doi: <http://dx.doi.org/I0.5569/23405IO4.03.OI.I2> 


\section{Responsabilidad social empresarial}

La responsabilidad social empresarial (en adelante RSE) expresa la voluntad de la empresa de aportar a la sociedad contribuciones positivas -adicionales a las exigidas por la ley- y a las que constituyen su responsabilidad básica de creación de riqueza (Blanco, 20I0). De este modo las empresas, conscientes del origen de sus activos económicos, podrán reinvertir parte de sus beneficios en el desarrollo de otros aspectos humanos, sociales y medioambientales que no le son ajenos, satisfaciendo con ello las expectativas de sus principales grupos de interés o stakeholders (Carroll, I993; Freeman, I984; Lozano, 2002; Verbeke y Tung, 2013).

El Libro Verde para Fomentar un marco europeo para la responsabilidad social de las empresas (Comisión de las Comunidades Europeas, 200I: 5 ) recuerda que la RSE puede contribuir de forma positiva al objetivo del Consejo Europeo de Lisboa de desarrollar una economía competitiva, dinámica y socialmente cohesionada. Una economía que potencie “prácticas idóneas en relación con la formación continua, la organización del trabajo, la igualdad de oportunidades, la integración social y el desarrollo sostenible”. Para ello, el documento de la Comisión destaca que las prácticas responsables de una empresa en materia social afectan tanto a una dimensión externa como interna y, dentro de ésta, en especial a la inversión en sus recursos humanos, la gestión del cambio y el desarrollo social en entornos dinámicos y competitivos.

En el ámbito específico de la gestión de personas, el Marco Promocional de la RSE elaborado por la Comisión de RSE de la Confederación Española de Organizaciones Empresariales (CEOE, 2006) identifica ocho actuaciones o buenas prácticas destinadas a los empleados como grupo de interés, entre las que podemos subrayar cuatro por su evidente relación con la integración laboral de colectivos vulnerables: a) respeto a su dignidad; b) respeto al principio de no discriminación; c) mejora de colectivos específicos con dificultad para la integración en el mercado de trabajo; y d) contribución a la empleabilidad de los trabajadores. Estos dos últimos apartados, de carácter voluntario al superar las exigencias legislativas vigentes, están directamente relacionados con la formación en prácticas y el empleo de personas con discapacidad intelectual.

\section{Empleo con apoyo de personas con discapacidad intelectual}

Aunque puedan presentarse incentivos económicos a la contratación de determinados grados de discapacidad, las ofertas de empleo para personas con discapacidad intelectual (que deban vincularse a programas de empleo con apoyo), revisten un carácter voluntario más allá del Real Decreto Legislativo I/20I3, que aprueba el texto refundido de la Ley General de Derechos de las Personas con Discapacidad y de su Inclusión Social. Por este motivo, el empleo con apoyo (en adelante EA) podría considerarse una práctica de RSE -y más específicamente cuando hacemos referencia a personas con mayores dificultades de integración sociolaboral, como puedan ser solicitantes de empleo con Síndrome de Down o personas con inteligencia límite-.

El EA hace referencia a la integración laboral de personas con discapacidad en empresas normalizadas gracias al "apoyo" de un preparador que acompaña al nuevo trabajador en el puesto de trabajo hasta lograr su autonomía e integración, supervisando posteriormente su evolución en la empresa (Kamp y Lynch, 2003). Por ello podríamos destacar que se trata de un sistema de refuerzo para personas que necesiten un seguimiento laboral individualizado en empresas ordinarias; seguimiento y apoyo que habría de ir retirándose progresivamente o bien espaciándose, hasta alcanzar un grado de autonomía satisfactorio por parte del trabajador (Verdugo et al, 1998). 


\section{El programa de integración de la Fundación Alimerka}

Alimerka es un grupo empresarial asturiano que cuenta con una red de I77 supermercados en Asturias, Castilla y León y Galicia. Con interés de centralizar la obra social de la empresa e iniciar nuevos proyectos de carácter socio comunitario, se constituyó la Fundación homónima en el año 2003 (BOPA I77, 3 I de julio de 2003).

Entre los años 2005 y 2014 la Fundación Alimerka coordinó el programa para facilitar la incorporación de personas con discapacidad intelectual en las empresas del Grupo, diseñando un protocolo con seis fases de trabajo para orientar los pasos a seguir por los diferentes implicados ${ }^{2}$ :

- Fase I. Identificación de los puestos de trabajo y los contextos adecuados. A partir de los análisis de puestos se identifican las funciones que podrán ser desempeñadas por personas con discapacidad intelectual. También se valoran los contextos adecuados: aspectos como número de empleados en cada supermercado o departamento, número de clientes diarios, proximidad a la asociación que prestará el apoyo, o acceso a medios de transporte, serán considerados para elegir los puestos y contextos más adecuados para la inserción.

- Fase 2. Contacto con las organizaciones sociales. Cuando surge una oportunidad laboral, se contacta con la organización de personas con discapacidad más próxima, solicitando su participación en el programa de EA y estableciendo un convenio de colaboración.

- Fase 3. Selección y formación de candidatos. La asociación correspondiente selecciona los

2. El vídeo titulado "Integración Laboral", disponible en el canal YouTube de Fundación Alimerka, resume el programa de empleo con apoyo desarrollado en la cadena de supermercados Alimerka. candidatos acordes con el perfil del puesto e imparte la formación para el empleo adecuada, combinando el desarrollo de habilidades sociales (saludo y despedida a clientes, petición de información a compañeros, etc.), con el conocimiento del producto o servicio, y el desarrollo de aptitudes específicas para el puesto (por ejemplo preparación de bandejas para horneado o colocación del producto en el punto de venta). En esta fase se considera esencial la colaboración de las familias, que deben conocer las características del EA y las condiciones laborales de la empresa, solicitando su consentimiento para continuar con el programa.

- Fase 4. Formación y sensibilización de los compañeros de trabajo. Se organizan reuniones entre representantes de las asociaciones y supervisores o mandos de la empresa para describir el programa, resolver dudas y eliminar posibles prejuicios y estereotipos que los futuros compañeros de trabajo pudiesen tener sobre el nuevo empleado.

- Fase 5. Acogida y empleo con apoyo. Cada joven contratado estará acompañado por un preparador laboral hasta que alcance autonomía en el puesto de trabajo. Este profesional será un referente estable tanto para el nuevo trabajador como para su familia y compañeros. En la elaboración de las adaptaciones necesarias al puesto de trabajo, el preparador prestará especial atención a la captación de "apoyos naturales”, identificado a quienes muestren más empatía y creen un clima laboral positivo e integrador para el trabajador.

- Fase 6. Seguimiento y evaluación. La implantación de un programa de estas características exige un seguimiento y una evaluación sistemática. La evaluación se establece ante tres hechos: el desempeño laboral del trabajador, la percepción que la empresa posee del resultado de la incorporación al puesto de trabajo y la actitud que mantiene el trabajador. 


\section{Resultados del programa}

El programa desarrollado ha facilitado el acceso al mundo laboral de I 7 jóvenes (diez personas con Síndrome de Down y siete con inteligencia límite), de los cuales nueve disfrutan de contratación indefinida. Por otro lado, en el programa de prácticas se oferta una media de diez plazas anuales en diferentes puestos.

La edad de estos jóvenes está comprendida entre los I9 y los 32 años, reconocidos con diferentes grados de discapacidad entre el $33 \%$ y el $68 \%$. El $48 \%$ son mujeres y el $52 \%$ hombres, residiendo el $63 \%$ en Asturias y el $37 \%$ en Castilla y León (León y Valladolid).

Para el seguimiento del programa se considera una inserción satisfactoria aquella que, pasados como mínimo dos años desde la finalización del EA, la persona sigue manteniendo su puesto de trabajo, desempeña adecuadamente las funciones del mismo y muestra indicadores de autonomía personal y social superiores a los manifestados antes de su incorporación al mundo laboral normalizado.

Respecto a la evaluación, se han empleado dos técnicas complementarias: un cuestionario a compañeros y supervisores de los trabajadores con discapacidad intelectual, y encuentros anuales con miembros de las asociaciones implicadas. La experiencia y los resultados hasta la fecha son satisfactorios, tanto desde el punto de vista laboral como social, valoración que también podemos extender hacia los clientes y, especialmente, las familias de los trabajadores, que manifiestan haber detectado un incremento de la autonomía, integración social y satisfacción personal de sus hijos o hermanos.

Para los encuentros anuales, la Fundación Alimerka ha coordinado ocho jornadas con responsables y técnicos de empleo de las Asociaciones Down de Asturias, León y Valladolid, la Fundación Edes y la Asociación Emburria. En estas jornadas, celebradas entre
2006 y $2014^{3}$, se ha podido reflexionar sobre el trabajo realizado en el marco del EA, así como fomentar el conocimiento personal de los preparadores de diferentes provincias y valorar el desarrollo del programa, identificando posibles cambios o actualizaciones en el diseño de puestos.

En las dos primeras jornadas el mayor peso lo tuvieron los miembros de las juntas de gobierno de las asociaciones implicadas, que debatieron sobre la forma idónea de articular las relaciones entre la empresa y las asociaciones de personas con discapacidad intelectual. A partir de 2008, el enfoque de las jornadas fue orientándose hacia una óptica más técnica, enfatizando el protagonismo de los preparadores laborales y mandos de la empresa. A partir de 20I2, también se invitó a participar a los jóvenes contratados y en prácticas, ofreciéndoles la oportunidad de evaluar el programa de empleo desde su mirada como personas con discapacidad ${ }^{4}$.

El análisis de contenido de las actas de las jornadas técnicas, donde se recogen las intervenciones de los diferentes participantes -gestores, preparadores laborales, trabajadores con discapacidad y supervisores-, permite identificar conclusiones que podríamos agrupar en tres áreas de trabajo: el de gestión de asociaciones, el de orientación y preparación laboral, y el de gestión de recursos humanos en la empresa contratante. Estas conclusiones se numeran a continuación:

\section{Aspectos a valorar desde la gestión de las Asociaciones de personas con discapacidad}

I. Es necesario implicar a las familias en los procesos de integración laboral. Para

\footnotetext{
3. I ${ }^{\mathrm{a}}$ Jornada técnica, celebrada el I 2/06/2006 en Down León; $2^{a}$ Jornada técnica: 23/10/2007, en Down León; $3^{a}$ Jornada: I 5/04/2008, en Down León; 4 J Jornada: 31/05/2009, en Centro Logístico Alimerka; 6 a Jornada: 29/I I/I 2, en Centro Logístico Alimerka; $7^{\mathrm{a}}$ Jornada: 28/05/2013, en Asociación Emburria, Cangas de Onís; 8 a Jornada: ०8/07/20I4, en Down León.

4. Un resumen de una de estas jornadas puede encontrarse en el canal YouTube de Fundación Alimerka, bajo el título "Jornada de Empleo con Apoyo - Cangas de Onís 2013”.
} 
motivar a las familias es adecuado que las asociaciones "rompan" esquemas y prejuicios de los padres, organizando pequeños talleres y potenciando la difusión de la integración laboral tanto a nivel asociativo como a través de los medios de comunicación.

2. El equipo directivo debe sensibilizar a las familias sobre la importancia del contenido del trabajo en vez de la imagen social del mismo. En este caso, los trabajos de “cuello azul” pueden llegar a ser, según las funciones y tipo de empresa, más complejos que otros trabajos de "cuello blanco", tanto desde un punto de vista cognitivo como manipulativo. En vez de prejuzgar una oferta laboral por su status asociado, debe valorarse la descripción del puesto, funciones o relaciones sociales que se establecen.

3. Debe coordinarse el trabajo entre tres unidades: empresa-asociación-familia. La empresa debe implicarse tanto con la asociación como con la familia, del mismo modo que la familia debe aceptar su vínculo tanto con la empresa como con la asociación.

4. El organigrama de cada asociación repercute directamente en los programas de empleo. En aquellas organizaciones en las que los preparadores laborales y técnicos poseen más autonomía y flexibilidad de trabajo resulta más eficiente la incorporación del trabajador y la integración del triángulo familia-empresaasociación.

\section{Aspectos a valorar desde los equipos de empleo}

I. El preparador laboral deberá conocer el puesto de trabajo que será ocupado, desempeñando previamente unas horas o unos días las principales actividades del puesto para luego poder trasmitirlas.
2. El preparador debe acompañar al trabajador a visitar su futuro centro de empleo unos días antes de su incorporación. Como el preparador ya conocerá la empresa ejercerá de “anfitrión” junto con la persona encargada del centro de trabajo.

3. Para vincular e incentivar a los futuros compañeros de trabajo, conviene hacer una presentación que responda a tres interrogantes: ¿qué es la discapacidad intelectual?, ¿qué características tiene un trabajador con síndrome de Down / inteligencia límite / etc.? y ¿qué función cumple el EA?

4. Además de la propia incorporación laboral, los preparadores pueden organizar grupos de autogestión del ocio. De este modo, los asociados que ya estén trabajando pueden reunirse semanalmente con sus preparadores laborales para decidir y planificar su tiempo libre.

5. Sería adecuado disponer de un protocolo de EA que indicase pautas a seguir, competencias que deben trabajarse o estrategias para trabajar en el entorno de trabajo. Esto no debe hacernos olvidar la variabilidad de cada incorporación laboral ni la flexibilidad necesaria para adaptarse a cada situación.

6. El preparador debe preguntarse qué pasará cuando se vaya y, si tiene dudas, pensar en posibles acciones de apoyo y seguimiento.

7. El foro y el chat de Down España es una importante fuente de información y resolución de dudas para los preparadores laborales. También los encuentros periódicos entre preparadores laborales son muy útiles para contrastar puntos de vista $\mathrm{y}$ formas de trabajo diferentes.

\section{Aspectos a valorar por la empresa}

I. La empresa ha de definir su objetivo con la integración de trabajadores con 
discapacidad intelectual. Debe identificar si persigue una acción de RSE, una ganancia de reputación social, la cobertura de un puesto de trabajo o una fusión de todos estos objetivos.

2. En caso de necesitar la cobertura de un puesto de trabajo, es necesario insistir en la diversidad, pues cada trabajador y cada centro de trabajo tienen características específicas. Por tanto, algunos trabajadores con discapacidad intelectual serán un apoyo importante pero no podrán desempeñar con máxima eficacia todas las funciones de un puesto.

3. Es necesario reflexionar sobre los motivadores que la empresa ofrece a los compañeros del trabajador con discapacidad intelectual, pues éstos también sentirán "desgaste" por la interacción continua. Refuerzos como reconocer su papel de apoyo, y escuchar sus valoraciones pueden ser muy valorados.

4. Los trabajadores con discapacidad intelectual necesitan tener un "apoyo natural” o compañero de trabajo que sea su referente en la empresa. No debemos confundir jerarquía con apoyo natural, pues la empresa no debe imponer este rol a ningún encargado o trabajador y será la propia dinámica de las relaciones interpersonales la que hará que surjan vínculos afectivos.

5. Es muy importante la actitud de los compañeros y supervisores en el lugar de trabajo, por lo cual hay que seleccionar adecuadamente el destino para la incorporación, procurando evitar centros con alta rotación de empleados para poder conseguir apoyo natural.

\section{Conclusión}

La Fundación Alimerka ha gestionado entre 2005 y 2014 un programa de integración laboral en la empresa ordinaria, impulsando la contratación de I 7 jóvenes con Síndrome de Down o con inteligencia límite en puestos de ayudante de panadería y de reponedor en supermercados, así como gestionando prácticas de una media de ro personas al año con discapacidad intelectual.

Con ánimo de efectuar un seguimiento sistemático de este programa, durante ocho años se realizaron tanto cuestionarios como encuentros con todas las partes implicadas (gestores de las asociaciones y preparadores laborales, trabajadores con discapacidad y representantes de la empresa). Estos encuentros han sido determinantes para conocer las características del programa de incorporación laboral de personas con discapacidad intelectual, comprendiendo que su buena gestión implica un mayor compromiso que la mera contratación laboral.

La implicación conlleva la coordinación del trabajo con asociaciones o entidades de personas con discapacidad, así como un seguimiento del empleo con apoyo y la evaluación de sus resultados tanto laborales como sociales. Por ello podríamos defender que la integración laboral de personas con discapacidad intelectual supone un acto laboral acorde con la legislación vigente, pero también un reto y una práctica de RSE. Y es que los fines de toda acción de responsabilidad social en una empresa habrán de ser coherentes con los retos de la sociedad de la que forma parte, como el desempleo, la integración de personas con discapacidad o la igualdad de las personas. 


\section{Referencias bibliográficas}

Blanco, A. (2010): "Las aportaciones de la Sociología española a la Responsabilidad Social Empresarial". Revista Internacional de Sociología (RIS), 68: 577-602.

Carroll, A. (1993): Business \& Society - Ethics and Stakeholder Management, Ohio: South-Western Cengage Learning $\left(2^{\mathrm{a}} \mathrm{Ed}\right)$

Comisión de las Comunidades Europeas (200I): Libro Verde. Fomentar un marco europeo para la responsabilidad social de las empresas. $\mathrm{COM} / 200 \mathrm{I} / 366$ final, de I 8 de julio de $200 \mathrm{I}$ (en línea). <http://eur-lex.europa.eu/legalcontent/ES/TXT/?uri=CELEX:5200IDC0366,, acceso 8 de enero de 2015 .

Confederación Española de Organizaciones Empresariales (CEOE) (2006): Borrador del Marco Promocional de la Responsabilidad Social, de 4 de noviembre de 2006, Documento interno.
Freeman, R.E. (1984): Strategic Management: A Stakeholder Approach, Boston: Pitman.

Kamp, M. y Lynch, C. (2003): Handbook Supported Employment, Middelharnis, Netherlands: Stichting Zuidwester.

Lozano, J.M. (2002): “ ¿Hacia la empresa ciudadana?”, en Albarello, M. (coord.): La Responsabilidad Social Corporativa y los Códigos de Conducta: ¿̇eneficios para todo el mundo? Barcelona: Fundació Pau i Solidaritat de Comissions Obreres de Catalunya.

Verbeke, A. y Tung, V. (20I3): "The Future of Stakeholder Management Theory: A Temporal Perspective”. Journal of Business Ethics, vol. I I2 (3): 529-543.

Verdugo, M.A. et al. (I998): "Situación actual del empleo con apoyo en España”. Siglo Cero, I75, vol. 29: 23-3I. 